\title{
Pick's Disease
}

National Cancer Institute

\section{Source}

National Cancer Institute. Pick's Disease. NCI Thesaurus. Code C85008.

A rare neurodeg enerative disorder leading to dementia. It is characterized by frontotemporal lobar degeneration with accumulation of tau proteins which form Pick bodies. 